\title{
Palladium/silicon nanowire Schottky barrier-based hydrogen sensors
}

\author{
Karl Skucha*, Zhiyong Fan, Kanghoon Jeon, Ali Javey, Bernhard Boser \\ Department of Electrical Engineering and Computer Sciences, University of California at Berkeley, Berkeley, CA 94720, USA
}

\section{A R T I C L E I N F O}

\section{Article history:}

Received 25 September 2009

Received in revised form

16 November 2009

Accepted 27 November 2009

Available online 2 December 2009

\section{Keywords:}

Hydrogen sensor

Gas sensor

Schottky barrier

Nanowire

Hydrogen detection

$\mathrm{H}_{2}$ sensor

\begin{abstract}
A B S T R A C T
This work presents the design, fabrication, and characterization of a hydrogen sensor based on a palladium/nanowire Schottky barrier field-effect transistor that operates at room temperature. The fabricated sensor consists of boron-doped silicon nanowire arrays that are contact printed on top of a $\mathrm{SiO}_{2} / \mathrm{Si}$ substrate with subsequently evaporated Pd contacts. The fabrication process is compatible with post-CMOS and plastic substrate integration as it can be completed at temperatures below $150{ }^{\circ} \mathrm{C}$ with good yield and repeatability. The sensor can reliably and reversibly detect $\mathrm{H}_{2}$ concentrations in the range from $3 \mathrm{ppm}$ to $5 \%$ and has a sensitivity of $6.9 \% / \mathrm{ppm}$ at $1000 \mathrm{ppm}$. A response distinguishable from drift and noise is produced in less than $5 \mathrm{~s}$ for $\mathrm{H}_{2}$ concentrations over $1000 \mathrm{ppm}$ and less than $30 \mathrm{~s}$ for concentrations over $100 \mathrm{ppm}$. The sensor settles to $90 \%$ of the final signal value in about $1 \mathrm{~h}$ at lower concentrations and less than $1 \mathrm{~min}$ at $10,000 \mathrm{ppm} \mathrm{H}_{2}$. Drift over an 87-h measurement period is below $5 \mathrm{ppm}_{2}$ concentration. (c) 2009 Elsevier B.V. All rights reserved.
\end{abstract}

\section{Introduction}

Sensors based on carbon nanotubes (CNT) and semiconductor nanowires (NW) have been reported for the detection of various gases, chemicals, and biomolecules. Many of these sensors achieve excellent performance, attributed in part to the high surface to volume ratio of nanostructures [1-6]. Highly uniform sensor fabrication, however, has been a challenge in many of these efforts, especially those enabled by the bottom-up process. The solution presented here overcomes this problem by utilizing the statistical averaging from multiple parallel nanowires acting as one sensor element. The fabrication process is based on a NW contact printing technology described recently [7-9]. In this approach, NWs are grown in a CVD system on a donor substrate and are subsequently transferred to the sensor substrate. The separation of NW growth from sensor fabrication imposes minimum constraints on the growth process and the choice of substrates available for the sensors. Since printing is a room temperature process, it is compatible with a very wide selection of substrates including fabricated semiconductors and flexible materials such as plastics or paper [7]. The sensors presented here are fabricated on Si dies as a preliminary step towards fabricating arrays of NW sensors on active electronic substrates with embedded evaluation circuits.

\footnotetext{
* Corresponding author at: BSAC UC Berkeley, 497 Cory Hall \#1774, University of California, Berkeley, CA 94720-1774, USA. Tel.: +1 510643 6690; fax: +15106436637.

E-mail address: kskucha@eecs.berkeley.edu (K. Skucha).
}

This work presents contact printed silicon nanowires (SiNW) applied to high-sensitivity hydrogen gas detection. Hydrogen is used in many industrial processes such as hydrogenation, petroleum transformation, cryogenic cooling, and chemical production. However, because this gas is odorless, colorless, and flammable at concentrations over $4 \%$, it poses safety concerns and creates a need for effective $\mathrm{H}_{2}$ leakage sensors. These applications frequently demand sensors with a lower limit of detection (LOD) of several ppm to identify small leaks that may be located far from the detector. High sensitivity and low drift are also required to reliably meet this requirement. Other important requirements include fast response time, good linearity, long lifetime, insensitivity to environmental changes including temperature and humidity, selectivity over other gases, and room temperature operation.

Many $\mathrm{H}_{2}$ sensors reported to date are based on the selective absorption of $\mathrm{H}_{2}$ by palladium, which results in the reversible formation of palladium hydride $\left(\mathrm{PdH}_{x}\right)$. Several properties of $\mathrm{Pd}$, including its work function, conductivity, lattice constant, and optical properties are modulated by this absorption. $\mathrm{H}_{2}$ sensors rely on one or more of these effects to detect $\mathrm{H}_{2}$ gas dissolved in ambient air.

Several $\mathrm{H}_{2}$ nanosensors have been developed over the past decade that operate at room temperature and can be fabricated or assembled using a low temperature process. To compare the performance of these sensors, the sensitivity and LOD are reported. Sensitivity is defined as the percent change in a physical parameter (such as resistance, current, etc.) per additional ppm of $\mathrm{H}_{2}$. Hydrogen sensitive CNT transistors are created by evaporating or electrodepositing non-continuous Pd beads onto a CNT film 
[10-12]. Upon exposure to $\mathrm{H}_{2}$, the workfunction change of $\mathrm{Pd}$ causes an increase in resistance of the CNT. Kong et al. reported a sensor based on this principle with a LOD below $40 \mathrm{ppm}$ [11] while Mubeen et al. reported a sensitivity of $0.42 \% / \mathrm{ppm}$ up to $1000 \mathrm{ppm}$ $\mathrm{H}_{2}$ concentration [12]. Nano-cluster-based $\mathrm{H}_{2}$ sensors take advantage of the $\mathrm{H}_{2}$-induced lattice expansion (HILE) in the Pd lattice, which causes the metal particles to swell and consequently fill open junctions in the cluster, increasing its conductance. Their high surface area to volume ratio results in a response time under $1 \mathrm{~s}$, albeit at the expense of low sensitivity. Xu et al. reported a nanocluster-based sensor with $0.007 \% / \mathrm{ppm}$ sensitivity at $1000 \mathrm{ppm} \mathrm{H}_{2}$ concentration [13]. The reported LOD of $25 \mathrm{ppm}$ corresponds to only a $2 \%$ resistance change, less than the typical sensor drift in many situations. Pd NW resistors exploit the natural decrease of the conductance of Pd when it absorbs $\mathrm{H}_{2}$ gas, but they also suffer from low sensitivity. Yang et al. reported a sensitivity below $0.002 \% / \mathrm{ppm}$ at $1000 \mathrm{ppm} \mathrm{H}_{2}$ concentration and an LOD of $2 \mathrm{ppm}$ [14]. As before, the corresponding resistance change of $0.15 \%$ is difficult to detect due to intrinsic sensor drift and potential changes in ambient conditions.

The sensitivity and LOD of reported nanosensors is vastly inferior to Pd/bulk-semiconductor Schottky barrier-based sensors presented in literature. In 1981, Ruths et al. presented a $\mathrm{Pd} / \mathrm{SiO}_{2} / \mathrm{Si}$

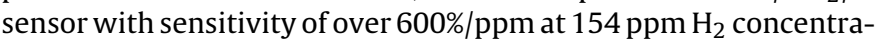
tion [15]. $\mathrm{Pd} / \mathrm{InP}$ and $\mathrm{Pd} / \mathrm{SiO}_{2} / \mathrm{AlGaN}$ diode-based sensors achieved even higher sensitivity of approximately $1000 \% / p p m[16,17]$. At $15 \mathrm{ppm}$, the reported response of over $3800 \%$ can be easily distinguished from drift and noise [16], suggesting that the LOD is significantly lower. Additionally, Schottky barrier-based sensors showed a nearly linear relationship between the sensor response and $\mathrm{H}_{2}$ concentration over a range from $15 \mathrm{ppm}$ to $1 \%$ [16].

The objective of this work is to combine the benefits of NW sensors, including their flexible manufacturing processes, with the high performance reported for Schottky-based bulk sensors. The proposed sensor is based on a Schottky barrier nanowire field-effect transistor (SBNWFET) with Pd contacts. Although Pd/Si sensors exhibit lower sensitivity than other semiconductors due to Fermi level pinning and undesired palladium silicide (PdSi) formation [15], SiNWs are used in this work because of their well-understood characteristics, tunability via doping or gate voltage, and welldeveloped growth and functionalization chemistries. Moreover, Si SBNWFETs have shown excellent electrical characteristics without needing the high temperature anneals usually required for good ohmic contacts in standard FETs [18].

Modulation of the Schottky barrier contacts by $\mathrm{H}_{2}$ has already been demonstrated for CNTs [19] and Wong et al. reported a Schottky barrier-based CNT $\mathrm{H}_{2}$ sensor, but it achieved a sensitivity several orders of magnitude lower than bulk sensors [20]. The NW sensor reported here achieves a drift limited LOD of only $5 \mathrm{ppm}$ and a sensitivity significantly exceeding that of nanosensors and several recently reported bulk diode designs [21-23].

\section{Sensing principle}

Fig. 1a shows the structure of the sensor consisting of a $\mathrm{Pd} / \mathrm{Si} / \mathrm{Pd}$ layers. As opposed to the standard metal-semiconductor architecture used in diode-based sensors, the metal-semiconductor-metal architecture was used here because it is simpler to fabricate (since an ohmic contact is not needed), and several other benefits, such as bi-directional sensing and potentially higher sensitivity [24]. A native $\mathrm{SiO}_{2}$ layer exists at each $\mathrm{Pd} / \mathrm{Si}$ interface and serves as a diffusion barrier against PdSi formation while concurrently reducing the effect of Fermi level pinning [15], but is too thin to inhibit carrier transport. As seen in Fig. 1b, under ambient conditions an ohmic contact forms between Pd and $\mathrm{Si}$. As $\mathrm{H}_{2}$ is introduced, it dissoci-


Fig. 1. (a) Schematic of the SBNWFET sensor. (b) Energy band diagram before $\mathrm{H}_{2}$ is introduced; holes see no barrier and the contact is ohmic. Native $\mathrm{SiO}_{2}$ layer is omitted in this diagram because it is too thin to affect transport. (c) Energy band diagram after hydrogen is introduced, showing a formation of a Schottky barrier.

ates into $\mathrm{H}^{+}$ions which thereafter diffuse into Pd and settle on the $\mathrm{Pd} / \mathrm{NW}$ interface. These ions induce a dipole layer and cause the workfunction of the metal to effectively decrease [25]. As seen in Fig. 1c, this eventually leads to the formation of a Schottky barrier which impedes holes from crossing over from the metal to the NW at the reverse biased source contact, which limits the current flow. If this current is primarily due to thermionic transport, it has an exponential dependence on the Schottky barrier given by:

$I_{S} \propto e^{-q \phi_{B} / k_{B} T}$

where $I_{S}$ is the current, $\phi_{B}$ is the Schottky barrier height (SBH), $k_{B}$ is the Boltzmann constant, $q$ is the electron charge, and $T$ is the temperature. Correspondingly, the change of the barrier height from ambient air to a certain $\mathrm{H}_{2}$ concentration at atmospheric pressure can be approximated with the Temkin model [26],

$\phi_{B}-\phi_{B_{0}}=\Delta \phi_{B}=V_{p} \ln \left(F \frac{\left[\mathrm{H}_{2}\right]}{\left[\mathrm{H}_{2}\right]_{0}}\right)$

where $V_{p}$ is a fitting parameter, $F$ is an equilibrium constant associated with the $\mathrm{H}^{+}$ion coverage on the $\mathrm{Pd} / \mathrm{SiO}_{2}$ interface, $\Delta \phi_{B}$ represents the change in barrier height, $\left[\mathrm{H}_{2}\right]$ is the $\mathrm{H}_{2}$ concentration and $\left[\mathrm{H}_{2}\right]_{0}$ is the intrinsic $\mathrm{H}_{2}$ concentration in ambient air, which is approximately $0.5 \mathrm{ppm}$. The parameter $V_{p}$ captures effects that depend on the method of Pd deposition [16] and the surface properties of the semiconductor. Substituting Eq. (1) into (2), and noting that sensor resistance is inversely proportional to the current for a constant bias $V_{d s}$, yields the sensor response:

$\frac{R_{f}-R_{0}}{R_{0}}=\frac{\Delta R}{R_{0}}=C \times\left[\mathrm{H}_{2}\right]^{n}$

where $R_{f}$ the resistance of the sensor at concentration $\left[\mathrm{H}_{2}\right], R_{0}$ is the resistance in ambient air, $C$ is a constant that equals $\left(F /\left[\mathrm{H}_{2}\right]_{0}\right)^{n}$ and $n=V_{p} / V_{T}$, where $V_{T}=k T / q$. This relationship indicates that the change in the resistance is dependent on a power of the $\mathrm{H}_{2}$ concentration. For $V_{p}=V_{T}$ the response is linear to first order. However, second order effects such as tunneling transport and the initial 




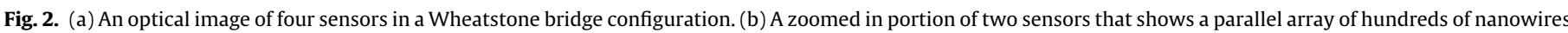
contacted between two Pd terminals.

ohmic region may cause $\Delta R / R_{0}$ to deviate from linearity. In addition, because of the finite number of interface states for $\mathrm{H}^{+}$ions [27], the sensor response saturates for $\mathrm{H}_{2}$ concentrations exceeding a few percent [26].

\section{Experimental}

\subsection{Fabrication}

SiNWs with an average diameter of $30 \mathrm{~nm}$ and a boron doping concentration of $10^{19} \mathrm{~cm}^{-3}$ were grown on a separate Si substrate using a vapor-liquid-solid (VLS) process. They were subsequently contact printed at room temperature on a second Si substrate with a $200 \mathrm{~nm} \mathrm{SiO}{ }_{2}$ on top that was pre-patterned with lift-off photoresist. A rinse in octane, acetone and IPA removed the resist, leaving regions of well-aligned, dense arrays of NWs. After printing of the NWs, the dies were placed in a petri dish and kept at room temperature in air with 40 to $55 \%$ humidity for at least one week to facilitate formation of approximately $8 \AA$ of native $\mathrm{SiO}_{2}$ [28]. A $5 \mathrm{~s}$ dip in 100:1 HF, used to remove contaminants from the NW surface, removed about 2-3 Å of this oxide [29], leaving the rest as a barrier against PdSi formation. This etch was followed immediately by the evaporation of $50 \mathrm{~nm}$ of Pd and a rapid thermal anneal for $30 \mathrm{~s}$ at $150^{\circ} \mathrm{C}$. The Pd contacts were made $200 \mu \mathrm{m}$ wide in order to reduce variability due to varying NW density. Although the average length of the printed NWs is typically over $20 \mu \mathrm{m}$, the contacts were separated by only $2 \mu \mathrm{m}$, ensuring that about $90 \%$ of all printed wires form contacts between the Pd junctions. Finally, the dies were assembled into standard IC packages and were wire bonded. Fig. 2 shows the completed sensor. The entire fabrication process

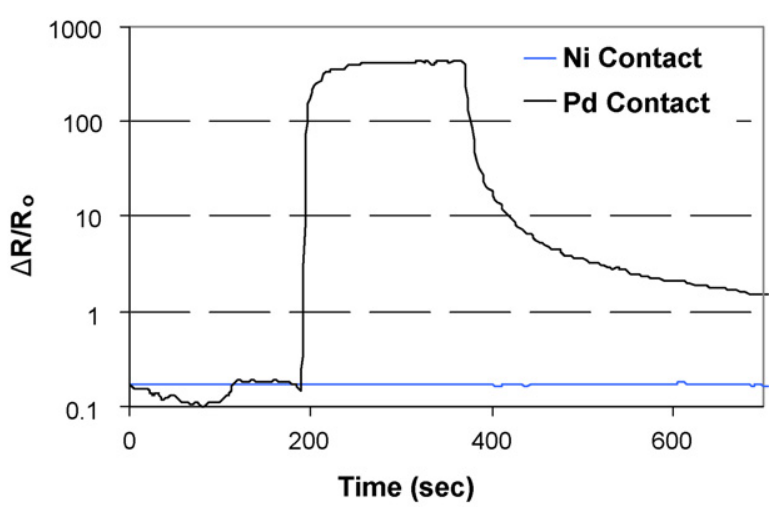

Fig. 3. Comparison of the responses of $\mathrm{Ni}$ and Pd contacted NWFETs to $1 \% \mathrm{H}_{2}$. requires only two lithographic steps to pattern the NW print area and the Pd contacts.

\subsection{Measurement setup}

All $\mathrm{H}_{2}$ sensing experiments were conducted in a 0.51 chamber by mixing the appropriate amounts of Praxair $5 \% \mathrm{H}_{2} / 95 \% \mathrm{~N}_{2}$ gas mixture and compressed air with a series of calibrated Dwyer VFA series flow meters and Wilkerson R03 series pressure regulators. The temperature and humidity in the chamber were maintained between $22-27^{\circ} \mathrm{C}$ and $18-23 \%$, respectively. An Agilent B1500A Semiconductor Device Analyzer was used to set the voltage bias and measure the current through the sensor.

\section{Sensor characterization and discussion}

\subsection{Sensing mechanism}

Several gases, such as $\mathrm{NO}_{2}$ and $\mathrm{NH}_{3}$, modulate the conductance of the NW by adsorbing to its surface and acting as oxidizing or reducing agents $[5,30]$. To ensure that the effect from $\mathrm{H}_{2}$ that is measured in this work stems primarily from the Pd/NW Schottky barrier modulation, and not a NW surface interaction, a second NWFET with nickel contacts was fabricated in the same process, except it was annealed at $450^{\circ} \mathrm{C}$ to form good ohmic contacts. Fig. 3 compares the response to $1 \% \mathrm{H}_{2}$ concentration of two NWFETs with either Ni or Pd contacts. The virtually absent response of the sensor with Ni contacts compared to that with Pd contacts confirms that the primary transduction mechanism is due to Schottky barrier modulation at the Pd/NW interface.

\subsection{Sensor bias}

Fig. 4 shows the current $I_{s}$ as a function of the drain voltage $V_{d s}$ for different $\mathrm{H}_{2}$ concentrations. As expected, the formation of a Schottky barrier decreases the conductance of the sensor as $\mathrm{H}_{2}$ is introduced. The response resembles that of a $\mathrm{H}_{2}$ sensitive diode in reverse bias, which generally achieves high sensitivity over a wider range than diodes biased in forward bias [16,23,24]. Since a SBNWFET has two back-to-back junctions, one of which is always reverse biased, the current is symmetric around $V_{d s}=0 \mathrm{~V}$. Modulating the backgate voltage can also be used to tune the sensitivity. Low backgate voltages, for example, increase the initial Schottky barrier height of the sensor and generally reduce the extent of the ohmic region, which is less sensitive to $\mathrm{H}_{2}$. At $V_{b g}=-20 \mathrm{~V}$, the maximum $\Delta R / R_{0}$ achieved at $1 \% \mathrm{H}_{2}$ was over 1400 , compared to approximately 500 at $V_{b g}=0 \mathrm{~V}$. Since a three order magnitude response is 


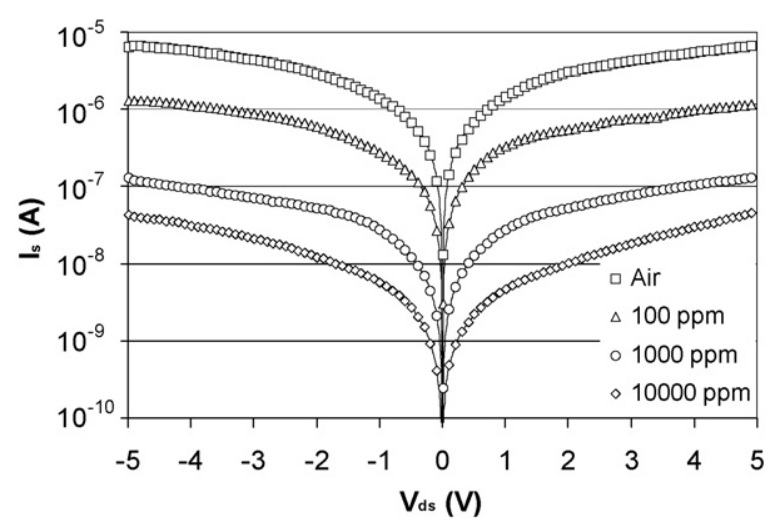

Fig. 4. $I_{s}$ vs. $V_{d s}$ at different $\mathrm{H}_{2}$ concentrations with $V_{b g}=0 \mathrm{~V}$.

not possible under tunneling transport at this NW's doping level, ${ }^{1}$ the current must be dominated by thermionic transport and hence our previous use of Eq. (1) is justified. In subsequent measurements the sensor is biased at $V_{d s}=2 \mathrm{~V}$, a value that represents a tradeoff between current and sensitivity, and $V_{b g}=0 \mathrm{~V}$, which maintains high sensitivity over a wide range and has a low drift.

\subsection{Sensitivity}

Fig. 5a shows the measured sensor response as a function of $\mathrm{H}_{2}$ concentration. The sensor response is reversible and does not saturate for $\mathrm{H}_{2}$ concentrations up to $5 \%$. Fig. 5b shows the responses to two consecutive $3 \mathrm{ppm} \mathrm{H}_{2}$ pulses, which are clearly above the intrinsic sensor noise. To establish the relation between the electrical sensor output and measured $\mathrm{H}_{2}$ concentration, the data from Fig. $5 \mathrm{a}$ is fitted to the model specified by Eq. (3), yielding $n=0.71$ and $C=13,100$. The result is shown in Fig. $5 c$ and is in excellent agreement with the model for concentrations in the range from $13 \mathrm{ppm}$ to $1 \%$. For lower concentrations the sensor is in the ohmic region of operation, whereas at $5 \%$ it approaches saturation. Measured data from two additional sensors tested confirm the power law, yielding a similar fit with values for $n$ of 0.71 and 0.79 and values for $C$ of 12,000 and 13,700 , respectively.

Sensitivity $S$ of the sensor, defined as

$S=\frac{d\left(\Delta R / R_{0}\right)}{d\left(\left[\mathrm{H}_{2}\right]\right)}=n C \times\left[\mathrm{H}_{2}\right]^{n-1}$

is $6.9 \% / \mathrm{ppm}$ at $1000 \mathrm{ppm}$, while for the other two sensors it is $6.3 \% / \mathrm{ppm}$ and $4.6 \% / \mathrm{ppm}$, respectively. This represents more than one order-of-magnitude improvement over the best result reported previously by $\mathrm{H}_{2}$ nanosensors based on other sensing principles.

\subsection{Response time}

Fig. 6 shows the response of the sensor to an increase of the $\mathrm{H}_{2}$ concentration from ambient to $13 \mathrm{ppm}$. After a fast initial transient, the output changes very slowly. Common measures such as $90 \%$ settling time require knowledge of the final value the output settles to. An accurate measurement of this value would take several hours and exceeds the drift caused by our experimental setup. Instead we found that the data is well modeled with the sum of two

\footnotetext{
${ }^{1}$ Tunneling probability at a boron doping level of $10^{19} \mathrm{~cm}^{-3}$ decreases by less than five times per $1 \mathrm{~V}$ of Schottky barrier change, about the maximum possible in $100 \% \mathrm{H}_{2}$ conditions. The response of our sensor is over two orders of magnitude higher at $1 \% \mathrm{H}_{2}$.
}

exponentials of the form

$$
\frac{\Delta R(t)}{R_{0}}=\frac{\Delta R_{\text {final }}}{R_{0}}\left[1-a e^{-t / \tau_{a}}-(1-a) e^{-t / \tau_{b}}\right]
$$

where $\tau_{a}$ and $\tau_{b}$ are first and second order time constants, respectively, and $a$ is a fitting parameter. Fig. 7 shows a plot of $\tau_{a}$ and $\tau_{b}$ obtained from fitting this model to measured data at concentrations from $3 \mathrm{ppm}$ to $5 \% \mathrm{H}_{2}$ concentration. The time for the signal to settle to $50 \%$ and $90 \%$ of the final value based on this fit are also shown. Since $a \approx 0.5$, independent of the $\mathrm{H}_{2}$ concentration, the settling time to $50 \%$ is dominated by $\tau_{a}$ while the much slower $\tau_{b}$ dominates settling to $90 \%$ of the final value. At low concentrations, the $90 \%$ settling time is on the order of an hour and decreases to below $1 \mathrm{~min}$ at $1 \%$, comparable to the times reported for bulk diode sensors [16,17].

The relevant sensor response time strongly depends on the application. Whereas in some industrial process control applications the exact $\mathrm{H}_{2}$ concentration is needed, for leak detection it is often sufficient to report a sudden rise. Fig. 8 reports the time required for the sensor output to reach $\Delta R / R_{0}=0.95$, a value that corresponds to $5 \mathrm{ppm} \mathrm{H}_{2}$ and is clearly above the noise and drift of this sensor. Concentrations above $25 \mathrm{ppm}$ are detected in less
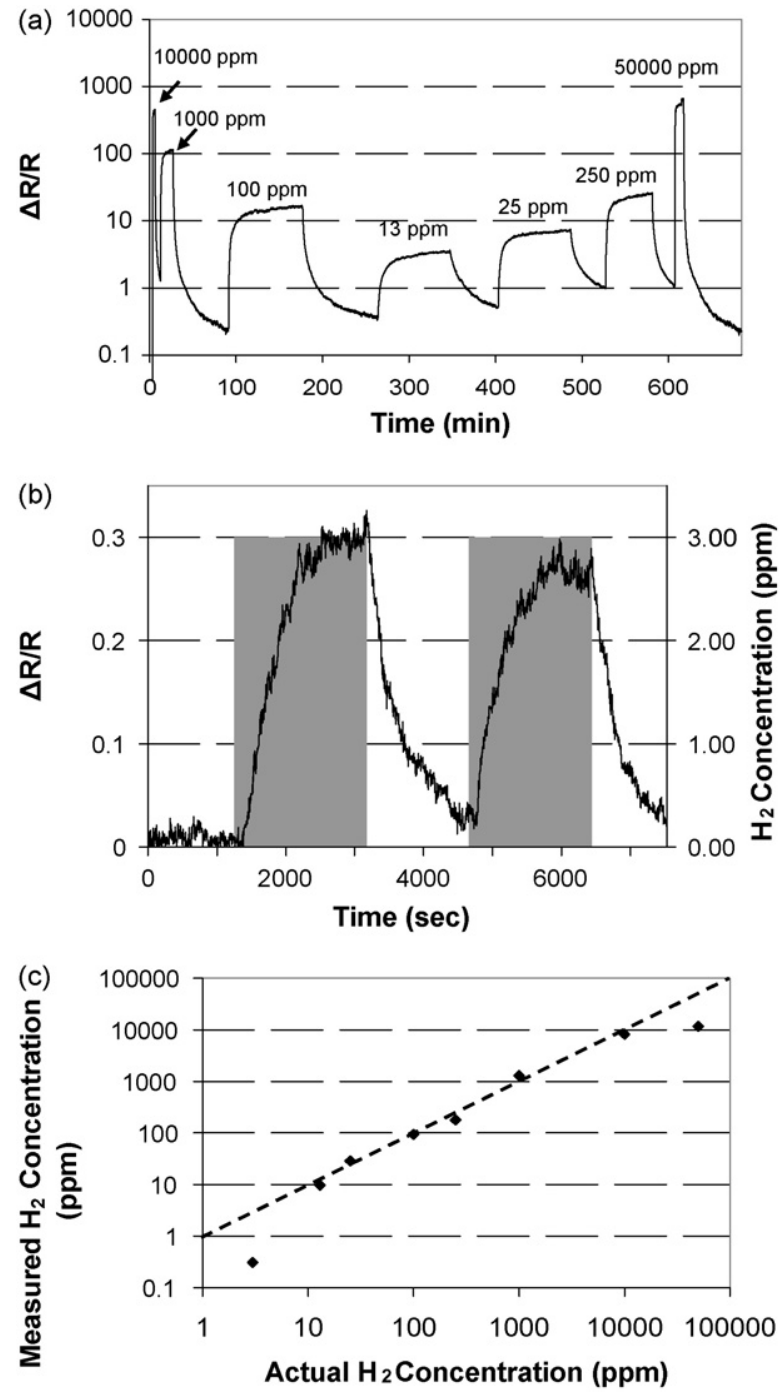

Fig. 5. (a) $\Delta R / R_{0}$ for $\mathrm{H}_{2}$ concentrations between $13 \mathrm{ppm}$ and $5 \%$ as a function of time. (b) Response to two consecutive pulses of $3 \mathrm{ppm} \mathrm{H}_{2}$ concentration. (c) Measured vs. actual $\mathrm{H}_{2}$ concentration. The dotted line represents 100\% accuracy. 
than $100 \mathrm{~s}$ while those above $1000 \mathrm{ppm}$ are detected in less than $5 \mathrm{~s}$.

Fig. 9 shows the recovery time of the $\mathrm{H}_{2}$ sensor, defined as the time the sensor settles to a resistance corresponding to a particular $\mathrm{H}_{2}$ concentration after exposure to $1 \% \mathrm{H}_{2}$ concentration. The $1 \mathrm{ppm}$ point, for example, corresponds to a recovery of approxi-

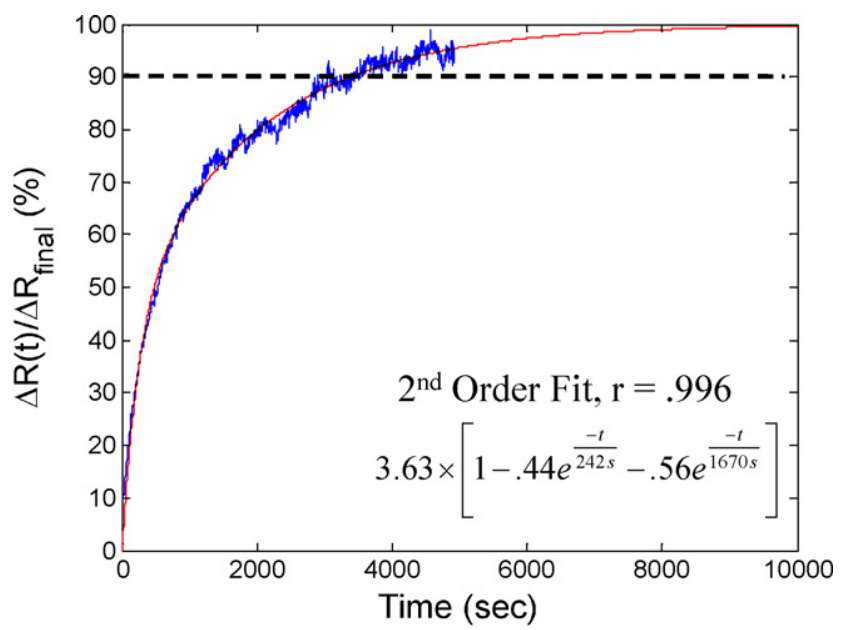

Fig. 6. Measured sensor response to $13 \mathrm{ppm}_{2}$ with fitted second order exponential model.



Fig. 7. First order time constant $\left(\tau_{a}\right)$, second order time constant $\left(\tau_{b}\right)$, and the $50 \%$ and $90 \%$ settling times vs. $\mathrm{H}_{2}$ concentration.

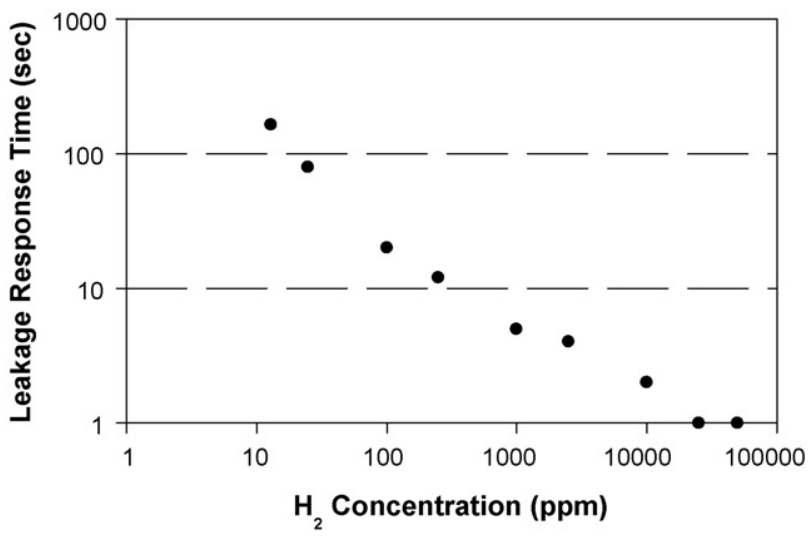

Fig. 8. Response time to a detectable leak vs. applied $\mathrm{H}_{2}$ concentration. A detectable leak is defined as the $5 \mathrm{ppm} \mathrm{H}_{2}$ level, which corresponds to a $\Delta R / R_{0}$ of $95 \%$. For concentrations above $2 \%$, the actual response time is less than $1 \mathrm{~s}$; the reported value is limited by the resolution of the measurement.

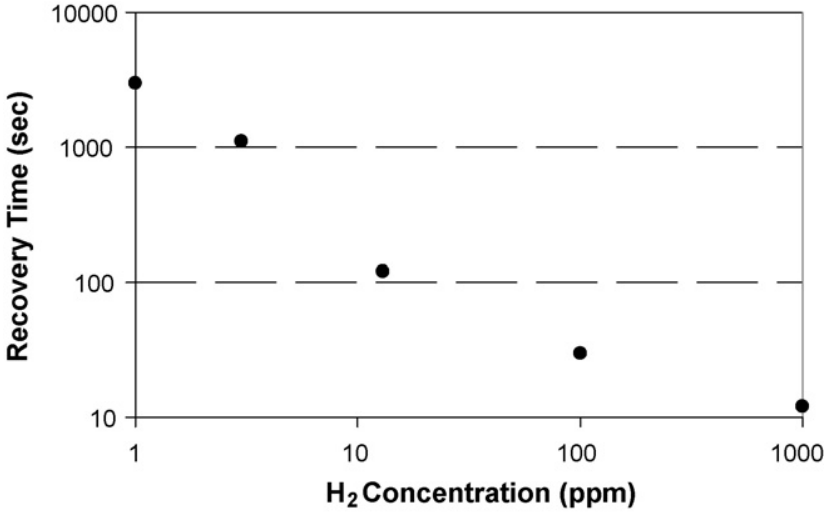

Fig. 9. Sensor recovery time from exposure at $1 \% \mathrm{H}_{2}$ to $\Delta R / R_{0}$ values corresponding to various $\mathrm{H}_{2}$ concentrations. The $1 \mathrm{ppm}$ concentration point corresponds to a recovery of approximately $10 \%$ within $R_{0}$.

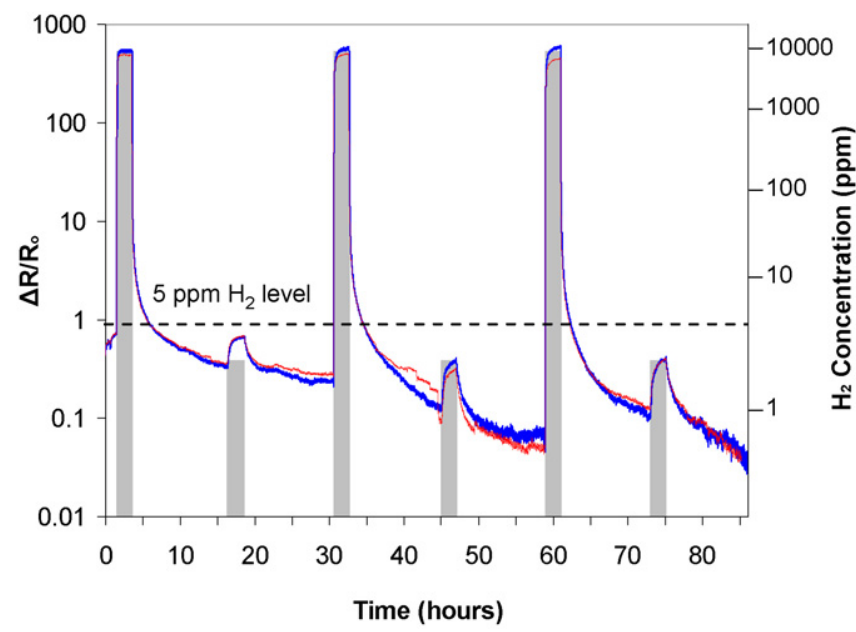

Fig. 10. $\Delta R / R_{0}$ vs. time for two sensors measured over a time span of $87 \mathrm{~h}$. At all times the $5 \mathrm{ppm} \mathrm{H}_{2}$ level is above the sensor drift.

mately $10 \%$ within $R_{0}$. Depending on the resolution requirement for the application, the relevant response time varies from minutes to seconds. For example, in scenarios that need detection of $\mathrm{H}_{2}$ concentrations of $100 \mathrm{ppm}$ and above, the relevant recovery time is less than $30 \mathrm{~s}$.

We have observed that response and recovery times are strongly affected by humidity and other contaminants, such as sulfur and carbon monoxide, present in the compressed air that was used. These contaminants are known to degrade response and recovery times of Pd-based $\mathrm{H}_{2}$ sensors [15,31]. In order to remove most of these impurities, sensors were heated to $150^{\circ} \mathrm{C}$ for $1 \mathrm{~min}$ in $\mathrm{N}_{2}$ ambient before each measurement was performed. We found this improved response times and recovery times by as much as 10 -fold in some cases.

\subsection{Drift}

To accurately measure the LOD and accuracy of the sensor in presence of drift and other effects, the sensor response was measured over an $87 \mathrm{~h}$ long period by cycling high and low concentrations of $\mathrm{H}_{2}$ in ambient air. The sensor was not heated before this experiment so that the amount of adsorbed water vapor and other contaminants stabilized. Fig. 10 shows an experiment in which two sensors are alternately exposed for two hours to $1 \%$ and $3 \mathrm{ppm} \mathrm{H}_{2}$, respectively. Each exposure is followed by a $12-\mathrm{h}$ 
recovery period and the entire sequence is repeated three times. Although drift at low concentrations is clearly visible, the sensor retains its ability to resolve $5 \mathrm{ppm}_{2}$ concentration. Despite this drift, the $1 \% \mathrm{H}_{2}$ responses exhibit variations below $\pm 10 \%$ in both sensors.

\subsection{Variability of the fabrication process}

To assess variations of sensor characteristics due to the fluctuations in the fabrication process, 14 sensors with identical dimensions were characterized. The value $R_{0}$ averaged $63.5 \mathrm{k} \Omega$ with a maximum variation of $\pm 40 \%$, though for sensors placed close together (in the same bridge configuration) the variation was only $\pm 20 \%$. These variations are low compared to single NW or single CNT based sensors, suggesting that the statistical averaging over many NWs in each sensor, enabled by the printing process, results in improved uniformity. All sensors were functional and achieved a $\Delta R / R_{0}$ at $1 \% \mathrm{H}_{2}$ of over 65 with an average of 160 and a maximum of 440 . The 5-s HF dip used during sensor fabrication to partially remove the native oxide has been found to be the major factor contributing to this variation.

\section{Conclusions}

NW contact printing is an attractive and flexible technique for bottom-up nanosensor fabrication. The use of multiple NWs for each sensor element reduces statistical variations arising from fluctuations in the fabrication process. A printed $\mathrm{H}_{2}$ sensor based on $\mathrm{Pd} /$ SiNW Schottky contacts achieves significantly higher sensitivity than nanosensors based on other sensing principles and enables reliable detection of $\mathrm{H}_{2}$ concentrations down to $5 \mathrm{ppm}$ due to its low drift. The printing process can be extended to other types of nanowire sensors either by functionalizing printed Si wires with receptors (as in the case for DNA or protein sensing) or by heterogeneously printing different types of nanowires that are naturally selective to other gaseous or chemical agents.

\section{Acknowledgements}

We acknowledge the support from Berkeley Sensor \& Actuator Center, Intel Corporation, and MARCO/MSD. K.R. Skucha is supported by the Intel Robert Byrd Fellowship.

\section{References}

[1] Y. Cui, Q.Q. Wei, H.K. Park, C.M. Lieber, Nanowire nanosensors for highly sensitive and selective detection of biological and chemical species, Science 293 (2001) 1289-1292.

[2] Z.Y. Fan, J.G. Lu, Gate-refreshable nanowire chemical sensors, Appl. Phys. Lett. 86 (2005) 123510-123512.

[3] E. Stern, J.F. Klemic, D.A. Routenberg, P.N. Wyrembak, D.B. Turner-Evans, A.D. Hamilton, D.A. LaVan, T.M. Fahmy, M.A. Reed, Label-free immunodetection with CMOS-compatible semiconducting nanowires, Nature (London) 445 (2007) 519-522.

[4] J. Kong, N.R. Franklin, C. Zhou, M.G. Chapline, S. Peng, K. Cho, H. Dai, Nanotube molecular wires as chemical sensors, Science 287 (2000) 622-625.

[5] M.C. McAlpine, H. Ahmad, D. Wang, J.R. Heath, Highly ordered nanowire arrays on plastic substrates for ultrasensitive flexible chemical sensors, Nat. Mater. 6 (2007) 379-384

[6] P. Chen, G. Shen, C. Zhou, Chemical sensors and electronic noses based on onedimensional metal oxide nanostructures, IEEE Trans. Nanotechnol. 7 (2008) 668-682.

[7] Z. Fan, J.C. Ho, Z.A. Jacobson, R. Yerushalmi, R.L. Alley, H. Razavi, A. Javey, Waferscale assembly of highly ordered semiconductor nanowire arrays by contact printing, Nano Lett. 8 (2008) 20-25.

[8] Z.Y. Fan, J.C. Ho, Z.A. Jacobson, H. Razavi, A. Javey, Large-scale, heterogeneous integration of nanowire arrays for image sensor circuitry, Proc. Nat. Acad. Sci. U. S. A. 105 (2008) 11066-11070.

[9] Z. Fan, J.C. Ho, T. Takahashi, R. Yerushalmi, K. Takei, A.C. Ford, Y.-L. Chueh, A. Javey, Towards the development of printable nanowire electronics and sensors, Adv. Mater. 21 (2009) 3730-3743.
[10] Y. Sun, H.H. Wang, Electrodeposition of Pd nanoparticles on single-walled carbon nanotubes for flexible hydrogen sensors, Appl. Phys. Lett. 90 (2007) 213107-213109.

[11] J. Kong, M.G. Chapline, H. Dai, Functionalized carbon nanotubes for molecular hydrogen sensors, Adv. Mater. 13 (2001) 1384-1386.

[12] S. Mubeen, T. Zhang, B. Yoo, M.A. Deshusses, N.V. Myung, Palladium nanoparticles decorated single-walled carbon nanotube hydrogen sensor, J. Phys. Chem. C 111 (2007) 6321-6327.

[13] T. Xu, M.P. Zach, Z.L. Xiao, D. Rosenmann, U. Welp, W.K. Kwok, G.W. Crabtree, Self-assembled monolayer-enhanced hydrogen sensing with ultrathin palladium films, Appl. Phys. Lett. 86 (2005) 203104-203106.

[14] F. Yang, D.K. Taggart, R.M. Penner, Fast, sensitive hydrogen gas detection using single palladium nanowires that resist fracture, Nano Lett. 9 (2009) 21772182.

[15] P.F. Ruths, S. Ashok, S.J. Fonash, J.M. Ruths, A study of Pd/Si MIS Schottky barrier diode hydrogen detector, IEEE Trans. Electron Devices 28 (1981) 10031009.

[16] Y.I. Chou, C.M. Chen, W.C. Liu, H.I. Chen, A new Pd-InP Schottky hydrogen sensor fabricated by electrophoretic deposition with Pd nanoparticles, IEEE Electron Device Lett. 26 (2005) 62-65.

[17] C.F. Chang, T.H. Tsai, H.I. Chen, K.W. Lin, T.P. Chen, L.Y. Chen, Y.C. Liu, W.C. Liu, Hydrogen sensing properties of a $\mathrm{Pd} / \mathrm{SiO}_{2} / \mathrm{AlGaN}$-based MOS diode, Electrochem. Commun. 11 (2009) 65-67.

[18] S.M. Koo, M.D. Edelstein, Q. Li, C.A. Richter, E.M. Vogel, Silicon nanowires as enhancement-mode Schottky barrier field-effect transistors, Nanotechnology 16 (2005) 1482-1485.

[19] A. Javey, J. Guo, Q. Wang, M. Lundstrom, H.J. Dai, Ballistic carbon nanotube field-effect transistors, Nature (London) 424 (2003) 654-657.

[20] Y.M. Wong, W.P. Kang, J.L. Davidson, A. Wisitsora-at, K.L. Soh, A novel microelectronic gas sensor utilizing carbon nanotubes for hydrogen gas detection, Sens. Actuators B: Chem. 93 (2003) 327-332.

[21] A. Salehi, A. Nikfarjam, D.J. Kalantari, Pd/porous-GaAs Schottky contact for hydrogen sensing application, Sens. Actuators B: Chem. 113 (2006) 419427.

[22] K.W. Lin, H.I. Chen, H.M. Chuang, C.Y. Chen, C.T. Lu, C.C. Cheng, W.C. Liu, Characteristics of Pd/InGaP Schottky diodes hydrogen sensors, IEEE Sens. J. 4 (2004) 72-79.

[23] Y.Y. Tsai, K.W. Lin, C.T. Lu, H.I. Chen, H.M. Chuang, C.Y. Chen, C.C. Cheng, W.C. Liu, Investigation of hydrogen-sensing properties of Pd/AlGaAsbased Schottky diodes, IEEE Trans. Electron Devices 50 (2003) 25322539.

[24] S.Y. Chiu, H.W. Huang, T.H. Huang, K.C. Liang, K.P. Liu, J.H. Tsai, W.S. Lour, Comprehensive investigation on planar type of Pd-GaN hydrogen sensors, Int. J. Hydrogen Energy 34 (2009) 5604-5615.

[25] A.Salomonsson, M. Eriksson, H. Dannetun, Hydrogen interaction with platinum and palladium metal-insulator-semiconductor devices, J. Appl. Phys. 98 (2005) 014505-014514.

[26] L.-G. Petersson, H.M. Dannetun, J. Fogelberg, I. Lundström, Hydrogen adsorption states at the external and internal palladium surfaces of a palladium-silicon dioxide-silicon structure, J. Appl. Phys. 58 (1985) 404-413.

[27] M. Eriksson, L.-G. Ekedahl, Hydrogen adsorption states at the $\mathrm{Pd} / \mathrm{SiO}_{2}$ interface and simulation of the response of a Pd metal-oxide-semiconductor hydrogen sensor, J. Appl. Phys. 83 (1998) 3947-3951.

[28] M. Morita, T. Ohmi, E. Hasegawa, M. Kawakami, M. Ohwada, Growth of native oxide on a silicon surface, J. Appl. Phys. 68 (1990) 1272-1281.

[29] K.R. Williams, K. Gupta, M. Wasilik, Etch rates for micromachining processingPart II, J. Microelectromech. Syst. 12 (2003) 761-778.

[30] X.T. Zhou, J.Q. Hu, C.P. Li, D.D.D. Ma, C.S. Lee, S.T. Lee, Silicon nanowires as chemical sensors, Chem. Phys. Lett. 369 (2003) 220-224.

[31] Z. Zhao, M.A. Carpenter, H. Xia, D. Welch, All-optical hydrogen sensor based on a high alloy content palladium thin film, Sens. Actuators B: Chem. 113 (2006) 532-538.

\section{Biographies}

Karl Skucha received with a B.S. degree in Electrical Engineering and Computer Sciences from University of California, Berkeley in 2006. He is an Intel Robert Noyce fellow and is currently completing his Ph.D. in Electrical Engineering at UC Berkeley. He is working with Prof. Bernhard Boser and Prof. Ali Javey on developing nanowire-based sensors and integrating them with active electronic circuits.

Zhiyong Fan received his B.S. and M.S. degrees in physical electronics from Fudan University, Shanghai, China, in 1998 and 2001, and Ph.D. degree in Materials Science from University of California, Irvine in December 2007. His Ph.D. research involved synthesis and characterization of nanowires for nanoelectronics and sensing applications. Currently he is a post-doctoral fellow in EECS of UC Berkeley, working on assembly and integration of semiconducting nanowires for nanoelectronics and energy harvesting applications.

Kanghoon Jeon received his B.S, degree in electrical engineering from Korea Advanced Institute of Science and Technology (KAIST), Daejon, Korea in 2006. He is currently pursuing a Ph.D. at University of California, Berkeley. His research interests include novel solid state device design for low power applications. 
Ali Javey is an assistant professor of electrical engineering and computer sciences at the University of California-Berkeley and a principal investigator in the Materials Sciences Division at Lawrence Berkeley National Laboratories. After receiving a Ph.D. in chemistry from Stanford University, he joined the faculty at UC Berkeley in 2005. His research interests focus on the integration of synthetic nano materials for various technological applications, including high-performance nanoelectronics, flexible circuits and displays, and novel electronic sensors.
Bernhard Boser received a Ph.D. from Stanford University in 1988. He was a Member of Technical Staff at Bell Laboratories. In 1992 he joined the faculty in the EECS Department of UC Berkeley. His research is in the area of analog and mixed signal circuits, with special emphasis on ADCs and micromechanical sensors and actuators. He has served on the program committees of ISSCC, VLSI Symposium, Transducers, is Vice President of the Solid-State Circuits Society and a Fellow of the IEEE. He is a co-founder of SiTime, a fabless semiconductor company. 